\title{
Effective Hausdorff dimension in general metric spaces
}

\author{
Elvira Mayordomo*
}

May 11, 2018

\begin{abstract}
We introduce the concept of effective dimension for a wide class of metric spaces that are not required to have a computable measure. Effective dimension was defined by Lutz in (Lutz 2003) for Cantor space and has also been extended to Euclidean space. Lutz effectivization uses the concept of gale and supergale, our extension of Hausdorff dimension to other metric spaces is also based on a supergale characterization of dimension, which in practice avoids an extra quantifier present in the classical definition of dimension that is based on Hausdorff measure and therefore allows effectivization for small time-bounds.

We present here the concept of constructive dimension and its characterization in terms of Kolmogorov complexity, for which we extend the concept of Kolmogorov complexity to any metric space defining the Kolmogorov complexity of a point at a certain precision. Further research directions are indicated.
\end{abstract}

\section{Introduction}

Effective dimension in Cantor space was defined by Lutz in [10, 11] in order to quantitatively study complexity classes [7]. The connections of effective dimension with Information Theory [14, in particular with Kolmogorov complexity and compression algorithms, some of them suspected even before the definition of effective dimension itself ([17, 18, 20, 21, 1, and more recently for symbolic dynamical systems in [19), have lead to very fruitful areas of research including those within Algorithmic Information theory 3 .

In this paper we will explore the definition of effective dimension for general metric spaces. The long term purpose of this line of research is to find more and easier dimension bound proofs in those spaces, while the connections with Information Theory already suggest further developments.

${ }^{*}$ Dept. de Informática e Ingeniería de Sistemas, Instituto de Investigación en Ingeniería de Aragón (I3A), Universidad de Zaragoza, Zaragoza, SPAIN. elvira@unizar.es. Part of this work was done during a visit to Iowa State University, supported by NSF Grants 1247051 and 1545028. 
The original definition of effective dimension was done in Cantor space which is the set of infinite binary sequences with the usual longest-common-prefixbased metric. The spaces of infinite sequences over other finite alphabets have also been explored, for instance the case of Finite-State effectivity is particularly interesting with this variation [2]. Also the Euclidean space $\mathbb{R}^{n}$ has been considered in this context by several papers that go back to fractal geometry, starting in [13] and including recent progress on the Kakeya conjecture [12], and the space $h^{\omega}$, a generalization of Cantor space, is studied in [15]. Our approach generalizes all the cited existing ones and does not require the underlying space to have a computable measure 1

Gales and supergales, introduced by Lutz in [10, are intuitively betting strategies in a guessing game on the elements of Cantor space. They allow the interpretation of Hausdorff dimension in terms of prediction and provide natural effectivizations of dimension by restricting the computability and resourcebounds used in the computation of these betting strategies. In terms of the complexity of the definition, the effectivization based on a gale characterization of dimension avoids an extra quantifier on the covers that would be present in effectivizations based on Hausdorff measure and makes it possible to attain low time-bounds effectivizations such as polynomial-time dimension.

We introduce here the concept of a nice cover of a metric space. A nice cover can simulate very closely any of the covers required in the definition of Hausdorff dimension, while it allows simple representations of the points in the space and the use of gales as betting games on those representations. Spaces with nice covers can be fairly general and are locally separable.

We then characterize Hausdorff dimension using supergales for any metric space with a nice cover. This characterization allows the definition of effective dimension by restricting the family of supergales that can be used.

We present here an initial step in this effectivization direction by introducing the definition of constructive dimension on a metric space. We then extend the concept of Kolmogorov complexity to any metric space and define the Kolmogorov complexity of a point at a certain precision. We characterize constructive dimension in terms of Kolmogorov complexity and prove the property of absolute stability (that is, the fact that constructive dimension can be pointwise defined). We present a few interesting examples of spaces with a constructive dimension including all previously studied cases. We finish with a list of topics for further development.

\section{Preliminaries}

In this section we include the definition of Hausdorff diemnsion for general metric spaces and include basic notation for strings and sequences.

\footnotetext{
${ }^{1}$ Several authors 5, 9, 16 have defined Martin-Löf algorithmical randomness in certain computable metric spaces, with the obvious requirement of a computable Borel measure that we avoid for effective dimension.
} 
Let $(X, \rho)$ be a metric space. (From now on we will omit $\rho$ when referring to space $(X, \rho))$.

Definition. The diameter of a set $A \subseteq X$ is

$$
\operatorname{diam}(A)=\sup \{\rho(x, y) \mid x, y \in A\} .
$$

Notice that the diameter of a set can be infinite.

Definition. Let $A \subseteq X$. A cover of $A$ is $\mathcal{C} \subseteq \mathcal{P}(X)$ such that $A \subseteq \cup_{U \in \mathcal{C}} U$.

Definition. Let $A \subseteq X$. $A$ is separable if there exists a countable set $S \subseteq A$ that is dense in $A$, that is, for every $x \in A, \delta>0$ there is an $s \in S$ such that $\rho(x, s)<\delta$.

Definition. The ball of radius $r>0$ about $x \in X$ is the set $B(x, r)=$ $\{y \in X \mid \rho(y, x)<r\}$.

Definition. A neighborhood of $x \in X$ is a set $U$ that contains a ball about $x$.

Definition. A locally separable space is $X$ such that every $x \in X$ has a neighborhood that is separable.

Definition. An isolated point in $X$ is $x \in X$ such that there is a $\delta>0$ with $B(x, \delta) \cap X=\{x\}$.

We will be interested in metric spaces that have no isolated points. Notice that metric spaces consisting only of isolated points have little interest for Hausdorff dimension (see definition below), while Hausdorff dimension in general spaces can be analyzed by restricting to non isolated points in the space.

We include the basic definitions of Hausdorff dimension. We refer the reader to [4] for a complete introduction and motivation.

For each $A \subseteq X$ and $\delta>0$, we define the set of countable $\delta$-covers of $A$

$\mathcal{H}_{\delta}(A)=\{\mathcal{U} \mid \mathcal{U}$ is a countable cover of $A$ and $\operatorname{diam}(U)<\delta$ for every $U \in \mathcal{U}\}$.

We can now define $H_{\delta}^{s}(A)$ and $H^{s}(X)$

$$
\begin{aligned}
H_{\delta}^{s}(A) & =\inf _{\mathcal{U} \in \mathcal{H}_{\delta}(A)} \sum_{U \in \mathcal{U}} \operatorname{diam}(U)^{s} . \\
H^{s}(A) & =\lim _{\delta \rightarrow 0} H_{\delta}^{s}(A) .
\end{aligned}
$$

Notice that $H_{\delta}^{s}(X)$ is monotone as $\delta \rightarrow 0$ so $H^{s}(X)$ is well defined. It is routine to verify that $H^{s}$ is an outer measure [4, $H^{s}$ is called the $s$-Hausdorff measure. Definition. (Hausdorff [6]). The Hausdorff dimension of $A \subseteq X$ is

$$
\operatorname{dim}_{\mathrm{H}}(A)=\inf \left\{s \in[0, \infty) \mid H^{s}(A)=0\right\} .
$$

Notice that $\operatorname{dim}_{\mathrm{H}}(A)$ is nontrivial only when $A$ is bounded, that is, $A$ has a finite diameter. In general there is an abuse of notation for unbounded $A$ using $\operatorname{dim}_{\mathrm{H}}(A)=\sup _{B \subseteq A, B}$ bounded $\operatorname{dim}_{\mathrm{H}}(B)$.

Let $\Sigma$ be a finite set. We denote as $\Sigma^{*}$ the set of finite strings over $\Sigma$ and $\Sigma^{\infty}$ the set of infinite strings over $\Sigma$. If $w \in \Sigma^{*}$ and $x \in \Sigma^{*} \cup \Sigma^{\infty}$ then $w \sqsubseteq x$ 
denotes $w$ being a finite prefix of $x$. For $0 \leq i \leq j$, we write $x[i \ldots j]$ for the string consisting of the $i$-th through the $j$-th symbols of $x$. We use $\lambda$ for the empty string. For $n \geq 0$, we write $x\lceil n$ for $x[0 . . n-1]$. ( $x\lceil 0$ denotes again the empty string).

We use $<,>$ for a pairing function $<,>: \Sigma^{*} \times \Sigma^{*} \rightarrow \Sigma^{*}$ that is injective, efficiently computable and invertible. We also denote with $\left\langle w_{1}, \ldots, w_{k}\right\rangle$ the $k$-fold composition of $<,>$ for each $k \in \mathbb{N}$.

\section{A supergale characterization of dimension in metric spaces}

\section{$3.1 \quad$ Nice covers}

We introduce the concept of a nice cover for a metric space. A nice cover allows well behaving representations of all points in the space, and it will be the key to the supergale characterization of Hausdorff dimension in the next subsection. Intuitively, a nice cover of $A$ is a sequence of covers of $A$ that can closely simulate any Hausdorff cover of $A$.

We prove here that spaces with a nice cover are locally separable and that spaces with countable nice covers are separable. We also include examples of spaces with nice cover that include all spaces for which effective dimension has been defined so far.

Our goal is to generalize Lutz characterization for Cantor space [10, 11]. Notice that Cantor space has very good properties not generally present in other spaces, namely a basis of clopen intervals defined by finite prefixes (for $\left.w \in \Sigma^{*}, C_{w}=\{x \mid w \sqsubseteq x\}\right)$ and a Borel measure from which the distance is defined $\left(\rho(x, y)=\inf \left\{\mu(w) \mid x, y \in C_{w}\right\}\right)$. These two facts make prediction and compression arguments natural and simpler, our aim is to have a prediction (and later on compression) setting for more general metric spaces.

Let $X$ be a metric space without isolated points.

Definition. A nice cover of $X$ is a sequence $\left(\mathcal{B}_{n}\right)_{n \in \mathbb{N}}$ with $\mathcal{B}_{n} \subseteq \mathcal{P}(X)$ for every $n$ and such that the following hold

(A1) (Decreasing monotonicity) For every $n \in \mathbb{N}, U \in \mathcal{B}_{n}, \mid\left\{V \in \mathcal{B}_{n+1}, V \subseteq\right.$ $U\} \mid<\infty$.

(A2) (Increasing monotonicity) For every $n \in \mathbb{N}, U \in \mathcal{B}_{n}, m<n$, there is a unique $V \in \mathcal{B}_{m}$ such that $U \subseteq V$.

(A3) (Layerwise nonnull) For every $n \in \mathbb{N}$, $\inf _{U \in \mathcal{B}_{n}} \operatorname{diam}(U)>0$.

(A4) (c-cover) There is a $c \in \mathbb{N}$ such that for every $A \subseteq X$ with $0<\operatorname{diam}(A)<$ 1 there exists $\left\{U_{1}, \ldots, U_{c}\right\} \subseteq \cup_{n} \mathcal{B}_{n}$ a cover of $A$, with $\operatorname{diam}\left(U_{i}\right)<c$. $\operatorname{diam}(A)$ for every $i$.

Notice that the above definition does not even require the elements of each cover $\mathcal{B}_{n}$ to be open or disjoint. 
Theorem 3.1 If $X$ has a nice cover then $X$ is locally separable.

Theorem 3.2 If $X$ has a countable nice cover then $X$ is separable.

We next include several examples of spaces with a nice cover including all spaces for which Hausdorff dimension has been effectivized. Our first example is the generalization of Cantor space to any finite alphabet and to any Borel probability measure.

Example 3.3 Consider the set $\Sigma^{\infty}$ of all infinite sequences over a finite alphabet $\Sigma$ with the metric based on a positive and nonatomic Borel probability measure.

Let $\nu: \Sigma^{*} \rightarrow[0,1]$ be a Borel probability measure on $\Sigma^{\infty}$ (that is, $\nu(\lambda)=1$ and for each $w \in \Sigma^{*} \nu(w)=\sum_{a \in \Sigma} \nu(w a)$ ) such that $\nu$ is positive (that is, $\nu(w)>0$ for every $\left.w \in \Sigma^{*}\right)$ and nonatomic (that is, $\lim _{n} \nu(x[0 . . n-1])=0$ for every $\left.x \in \Sigma^{\infty}\right)$. Then $\nu(w)$ is the measure of cylinder $\left.\mathbf{C}_{w}=\{x \in \mathbf{C} \mid w \sqsubseteq x\}\right)$, and the distance is defined as $\rho(x, y)=\inf \left\{\nu(w) \mid x, y \in \mathbf{C}_{w}\right\}$.

This space has a nice cover formed by cylinders $\mathbf{C}_{w}$. We take $\mathcal{B}_{n}=\left\{\mathbf{C}_{w} \mid w \in\{0,1\}^{n}\right\}$ for each $n \in \mathbb{N}$. (In this case condition (A4) is met for $c=1$ by considering the longest $w$ such that $A \subseteq \mathbf{C}_{w}$, we can prove that $\left.\operatorname{diam}(A)=\operatorname{diam}\left(\mathbf{C}_{w}\right)=\nu(w)\right)$.

Notice that we place no restrictions on the probability measure $\nu$, so this is a strict generalization of the case considered in [13], where $\nu$ needed to be "strongly positive" as defined in [13]. This is because of the good properties of Cantor space.

Particular cases of this metric space are widely used in connection with coding and compression algorithms, and also to represent symbolic dynamical systems.

We will now move to Euclidean space.

Example 3.4 For $m \in \mathbb{N}$, consider the set $\mathbb{R}^{m}$ with the metric based on a strongly positive Borel measure $\nu \in \Delta\left(\mathbb{R}^{m}\right)$.

We use the notation $I(\mathbf{z}, \mathbf{a}, n)\left(\mathbf{z} \in \mathbb{Z}^{m}, \mathbf{a} \in \mathbb{N}^{m}, n \in \mathbb{N}\right)$ for the n-diadic interval defined by $\mathbf{z}, \mathbf{a}$, that is, $I(\mathbf{z}, \mathbf{a}, n)=\left[z_{1}+a_{1} 2^{-n}, z_{1}+\left(a_{1}+1\right) 2^{-n}\right] \times$ $\ldots \times\left[z_{m}+a_{m} 2^{-n}, z_{m}+\left(a_{m}+1\right) 2^{-n}\right]$ (for every $i, a_{i}<2^{n}$ to avoid multiple representations).

$\nu$ is strongly positive if there is a $\delta>0$ such that $\nu(I(\mathbf{t}, \mathbf{b}, n+1))>\delta$. $\nu(I(\mathbf{z}, \mathbf{a}, n))$ for every $I(\mathbf{t}, \mathbf{b}, n+1) \subseteq I(\mathbf{z}, \mathbf{a}, n)$.

This space has a nice cover formed by intervals with dyadic rational extremes. We take $\mathcal{B}_{n}=\left\{I(\mathbf{z}, \mathbf{a}, n) \mid \mathbf{z} \in \mathbb{Z}^{m}, \mathbf{a} \in \mathbb{N}^{m}\right\}$ for each $n \in \mathbb{N}$. (In this case condition (A4) is met for $\left.c=\max \left(1 / \delta, 2^{m}\right)\right)$. Notice that an interval $[a, b]$ can be covered by two dyadic intervals $A, B$ of size $\nu(A) \leq \nu([a, b]) / \delta, \nu(B) \leq$ $\nu([a, b]) / \delta$.)

Example 3.5 Let $h: \mathbb{N} \rightarrow \mathbb{N}-\{0,1\}$, we define $h^{\omega}=\Pi_{n \in \mathbb{N}}\{0,1, \ldots, h(n)-1\}$ with the metric based on a positive and nonatomic Borel probability measure $\nu$.

For $n \in \mathbb{N}$, let $h^{n}=\Pi_{m<n}\{0,1, \ldots, h(m)-1\}$. Let $h^{*}=\cup_{n \in \mathbb{N}} h^{n}$. 
Given a Borel probability measure $\nu: h^{*} \rightarrow[0,1]$ (where $\nu(w)$ is the measure of cylinder $\left.\mathbf{C}_{w}=\left\{x \in h^{\omega} \mid w \sqsubseteq x\right\}\right)$, the distance is defined as $\rho(x, y)=$ $\inf \left\{\nu(w) \mid x, y \in \mathbf{C}_{w}\right\}$.

This space has a nice cover formed by cylinders $\mathbf{C}_{w}$. We take $\mathcal{B}_{n}=\left\{\mathbf{C}_{w} \mid w \in h^{n}\right\}$ for each $n \in \mathbb{N}$. (In this case condition (A4) is met for $c=1$ by considering the longest $w$ such that $A \subseteq \mathbf{C}_{w}$, we can prove that $\left.\operatorname{diam}(A)=\operatorname{diam}\left(\mathbf{C}_{w}\right)=\nu(w)\right)$.

This is a strict generalization of the case considered in [15].

Our last example has not been considered before in the context of effective dimension, and can have further interest in the context of spaces of functions.

Example 3.6 Let $n \in \mathbb{N}$. Let $P_{n}(0,1)$ be the set of polynomials with real coefficients and degree less than or equal to $n$, together with the metric $d(f, g)=$ $\|f-g\|_{\infty}=\sup _{x \in(0,1)}|f(x)-g(x)|$, for $f, g \in P_{n}(0,1)$.

This space has a nice cover defined from dyadic coefficient polynomials as follows. Let $\mathbf{z} \in \mathbb{Z}^{n+1}, \mathbf{a} \in \mathbb{N}^{n+1}, k \in \mathbb{N}$, with $a_{i}<2^{k}$ for every $i$,

$$
\left.D P(\mathbf{z}, \mathbf{a}, k)=\left\{b_{0}+b_{1} x+\ldots+b_{n} x^{n} \mid b_{i} \in \mathbb{R}, 0 \leq b_{i}-\left(z_{i}+a_{i} 2^{-k}\right) \leq 2^{-k}\right)\right\} .
$$

We take $\mathcal{B}_{k}=\left\{D P(\mathbf{z}, \mathbf{a}, k) \mid \mathbf{z} \in \mathbb{Z}^{n+1}, \mathbf{a} \in \mathbb{N}^{n+1}\right\}$ for each $k \in \mathbb{N}$. (In this case condition (A4) is met for $c=\max \left\{2^{n}, 2(n+1)\right\}$ ).

\subsection{Supergale characterization of Hausdorff dimension}

In this subsection we prove a supergale characterization of Hausdorff dimension for $X$ with a nice cover. Notice that each nice cover gives an equivalent characterization of dimension. The definition of a supergale in terms of diameters gives a new and interesting prediction setting for general metric spaces.

The concept of gale we introduce here is the natural extension of the gales introduced in [10 to spaces with nice covers, while the flexibility on the metric spaces makes the proof of this characterization quite more involved than the case of Cantor spaces proven in [10. For instance we cannot assume anything about the diameters of the covers used.

Let $X$ be a metric space with a nice cover, fix a nice cover $\left(\mathcal{B}_{n}\right)_{n \in \mathbb{N}}$. Let $\mathcal{B}=\cup_{n} \mathcal{B}_{n}$. For $n \in \mathbb{N}$, let $\mathcal{B}_{\geq n}=\cup_{m \geq n} \mathcal{B}_{m}$.

Definition. Given $x \in X$, a $\mathcal{B}$-representation of $x$ is a sequence $\left(w_{n}\right)_{n \in \mathbb{N}}$ such that $w_{n} \in \mathcal{B}_{n}$ and $x \in \cap_{n} w_{n}$.

We denote with $\mathcal{R}(x)$ the set of $\mathcal{B}$-representations of $x \in X$.

A supergale is intuitively a strategy in a betting game on a representation $\left(w_{n}\right)_{n \in \mathbb{N}}$ of an unknown $x \in X$.

Definition. Let $s \in[0, \infty)$. An $s$-supergale $d$ is a function $d: \mathcal{B} \rightarrow[0, \infty)$ such that the following hold

- $\sum_{U \in \mathcal{B}_{0}} d(U) \operatorname{diam}(U)^{s}<\infty$,

- for every $n \in \mathbb{N}$, for every $U \in \mathcal{B}_{n}$ the following inequality holds

$$
d(U) \operatorname{diam}(U)^{s} \geq \sum_{V \in \mathcal{B}_{n+1}, V \subseteq U} d(V) \operatorname{diam}(V)^{s} .
$$


An $s$-gale is an $s$-supergale for which equation (11) holds with equality.

Definition. An $s$-supergale $d$ succeeds on $x \in X$ if there is a $\left(w_{n}\right)_{n \in \mathbb{N}} \in \mathcal{R}(x)$, such that

$$
\limsup _{n} d\left(w_{n}\right)=\infty
$$

Definition. Let $d$ be an $s$-supergale. The success set of $d$ is

$$
S^{\infty}[d]=\{x \in X \mid d \text { suceeds on } x\} .
$$

Definition. $\hat{\mathcal{G}}(A)=\left\{s \mid\right.$ there is an $s$-supergale $d$ with $\left.A \subseteq S^{\infty}[d]\right\}$.

We start with two useful properties of supergales. The first one allows success to be defined independently of layers $\mathcal{B}_{n}$ for $n \in \mathbb{N}$, the second one is a generalization of Kraft inequality that is used in Cantor space, that gives us bounds on supergale values.

Property 3.7 Let $d$ be a an s-supergale, if there is a sequence in $\mathcal{B}\left(y_{m}\right)_{m \in \mathbb{N}}$ such that $\lim \sup _{m} d\left(y_{m}\right)=\infty$ then $\cap_{m} y_{m} \subseteq S^{\infty}[d]$.

Proof. From $\lim \sup _{m} d\left(y_{m}\right)=\infty$ and condition (1) in the definition of supergale we have that $\liminf _{m} \operatorname{diam}\left(y_{m}\right)=0$. In particular there is a subsequence $\left(y_{m_{k}}\right)_{k \in \mathbb{N}}$ with $\lim _{k} d\left(y_{m_{k}}\right)=\infty$ and $\lim _{k} \operatorname{diam}\left(y_{m_{k}}\right)=0$. Therefore using (A3) we have that for every $n$ there is a $k$ such that $y_{m_{k}} \in \mathcal{B}_{\geq n}$ which gives us $\left(w_{n}\right)_{n \in \mathbb{N}}$ such that $w_{n} \in \mathcal{B}_{n}$ with $\cap_{m} y_{m} \subseteq \cap_{n} w_{n}$ and $\lim \sup _{n} d\left(w_{n}\right)=\infty$.

Property 3.8 (Generalization of Kraft inequality) Let $d$ be an s-supergale. Then for every $\mathcal{E} \subseteq \mathcal{B}$ such that all sets in $\mathcal{E}$ are incomparable we have that

$$
\sum_{W \in \mathcal{B}_{0}} d(W) \operatorname{diam}(W)^{s} \geq \sum_{V \in \mathcal{E}} d(V) \operatorname{diam}(V)^{s} .
$$

Proof. Notice that $\sum_{U \in \mathcal{B}_{0}} d(U) \operatorname{diam}(U)^{s}<\infty$ implies that $d(U) \operatorname{diam}(U)$ is nonzero on a countable subset of $\mathcal{B}_{0}$, and therefore $d(U) \operatorname{diam}(U)$ is nonzero on a countable subset of $\mathcal{B}$ because of (A1). Therefore let us assume that $\mathcal{E} \subseteq \mathcal{B}$ is countable.

We prove that for any finite subset $\mathcal{A} \subseteq \mathcal{E}$,

$$
\sum_{W \in \mathcal{B}_{0}} d(W) \operatorname{diam}(W)^{s} \geq \sum_{V \in \mathcal{A}} d(V) \operatorname{diam}(V)^{s} .
$$

This can be proven by induction on $n$ such that $\mathcal{A} \subseteq \mathcal{B}_{\geq n}$ using condition (1) in the definition of supergale.

Our next Theorem is the supergale characterization of dimension. Since the metric used is not necessarily related to a Borel measure, and we can't assume a suitable basis of clopen (not even open) sets the arguments in the proof are different from the Cantor space case [10]. The fact that we need to use diameters instead of a probability measure makes it necessary to carefully examine each sum of diameters for its good definition and finiteness. 
Theorem 3.9 (Supergale characterization) Let $X$ be a metric space that has a nice cover, let $A \subseteq X$. Then

$$
\operatorname{dim}_{\mathrm{H}}(A)=\inf \hat{\mathcal{G}}(A) .
$$

\section{Proof.}

Let $s>\operatorname{dim}_{\mathrm{H}}(A)$. Then for any $k \in \mathbb{N}$ there is a countable cover of $A$, $\mathcal{C}_{k}$, such that $\sum_{U \in \mathcal{C}_{k}} \operatorname{diam}(U)^{s}<2^{-k}$ and $\operatorname{diam}(U)>0$ for each $U \in \mathcal{C}_{k}$. (If necessary substitute each $U_{n} \in \mathcal{C}_{k}$ with $\operatorname{diam}\left(U_{n}\right)=0$ by a ball of radius $\left.2^{-k / s-n / s-1}\right)$.

Using property (A4) of nice covers we can get a cover $\mathcal{E}_{k} \subseteq \mathcal{B}$ of $A$ such that

$$
\sum_{W \in \mathcal{E}_{k}} \operatorname{diam}(W)^{s}<c^{1+s} \cdot 2^{-k} .
$$

Let $\mathcal{D}_{k}=\left\{U \mid U \in \mathcal{E}_{k}\right.$ and no proper superset of $U$ is in $\left.\mathcal{E}_{k}\right\}$. Then $\mathcal{D}_{k}$ is a countable cover of $A$ and

$$
\sum_{W \in \mathcal{D}_{k}} \operatorname{diam}(W)^{s}<c^{1+s} \cdot 2^{-k}
$$

Define $d_{k}: \mathcal{B} \rightarrow[0, \infty)$ as follows,

For $U \in \mathcal{B}$, if $\operatorname{diam}(U)=0$ then $d(U)=1$.

If $U \in \mathcal{B}_{n}$ for $n>0$, $\operatorname{diam}(U)>0$, and there is $V \in \mathcal{B}_{n-1}$ and $W \in \mathcal{D}_{k}$ with $U \subseteq V \subseteq W, U \neq V$ and $\sum_{U^{\prime} \subseteq V, U^{\prime} \in \mathcal{B}_{n}} \operatorname{diam}\left(U^{\prime}\right)>0$ then

$$
d_{k}(U)=\frac{d_{k}(V) \operatorname{diam}(V)^{s}}{\sum_{U^{\prime} \subseteq V, U^{\prime} \in \mathcal{B}_{n}} \operatorname{diam}\left(U^{\prime}\right)^{s}} .
$$

Otherwise, if $U \in \mathcal{B}_{n}, \operatorname{diam}(U)>0$,

$$
d_{k}(U)=\sum_{W \in \mathcal{D}_{k} \cap \mathcal{B}_{\geq n}, W \subseteq U} \frac{\operatorname{diam}(W)^{s}}{\operatorname{diam}(U)^{s}} .
$$

Claim $3.10 d_{k}$ is an s-supergale.

Proof of Claim 3.10, Let $V \in \mathcal{B}_{n-1}$ and $\sum_{U^{\prime} \subseteq V, U^{\prime} \in \mathcal{B}_{n}} \operatorname{diam}\left(U^{\prime}\right)>0$.

If there is $W \in \mathcal{D}_{k}$ such that $V \subseteq W$ then

$$
\begin{aligned}
\sum_{U \subseteq V, U \in \mathcal{B}_{n}} d_{k}(U) \operatorname{diam}(U)^{s} & =\sum_{U \subseteq V, U \in \mathcal{B}_{n}} \frac{d_{k}(V) \operatorname{diam}(V)^{s}}{\sum_{U^{\prime} \subseteq V, U^{\prime} \in \mathcal{B}_{n}} \operatorname{diam}\left(U^{\prime}\right)^{s}} \operatorname{diam}(U)^{s} \\
& =d_{k}(V) \operatorname{diam}(V)^{s}
\end{aligned}
$$

If for any $W \in \mathcal{D}_{k}, V \nsubseteq W$ then

$$
d_{k}(V)=\sum_{W \in \mathcal{D}_{k} \cap \mathcal{B}_{\geq n-1}, W \subseteq V} \frac{\operatorname{diam}(W)^{s}}{\operatorname{diam}(V)^{s}} .
$$


Therefore,

$$
\begin{aligned}
\sum_{U \subseteq V, U \in \mathcal{B}_{n}} d_{k}(U) \operatorname{diam}(U)^{s} & =\sum_{U \subseteq V, U \in \mathcal{B}_{n}} \sum_{W \in \mathcal{D}_{k} \cap \mathcal{B}_{\geq n}, W \subseteq U} \frac{\operatorname{diam}(W)^{s}}{\operatorname{diam}(U)^{s}} \operatorname{diam}(U)^{s} \\
& =\sum_{U \subseteq V, U \in \mathcal{B}_{n}} \sum_{W \in \mathcal{D}_{k} \cap \mathcal{B}_{\geq n}, W \subseteq U} \operatorname{diam}(W)^{s} \\
& \leq \sum_{W \in \mathcal{D}_{k} \cap \mathcal{B}_{\geq n-1}, W \subseteq V} \operatorname{diam}(W)^{s}=d_{k}(V) \operatorname{diam}(V)^{s}
\end{aligned}
$$

where the last inequality follows from property (A2) of nice covers.

For every $U \in \mathcal{B}_{0}$, we use the second part in the definition of $d_{k}$. Therefore, using property (A2) of nice covers,

$$
\sum_{U \in \mathcal{B}_{0}} d_{k}(U) \operatorname{diam}(U)^{s} \leq \sum_{W \in \mathcal{D}_{k}} \operatorname{diam}(W)^{s}<c^{1+s} \cdot 2^{-k}<\infty .
$$

Claim 3.11 If $W \in \mathcal{D}_{k}, d_{k}(W)=1$.

Proof of Claim 3.11. If $W \in \mathcal{B}_{n}$, since all sets in $\mathcal{D}_{k}$ are incomparable, we use the second part in the definition of $d_{k}$ and

$$
d_{k}(W)=\sum_{W^{\prime} \in \mathcal{D}_{k} \cap \mathcal{B}_{\geq n}, W^{\prime} \subseteq W} \frac{\operatorname{diam}\left(W^{\prime}\right)^{s}}{\operatorname{diam}(W)^{s}}=1 .
$$

Claim 3.12 For every $k \in \mathbb{N}, U \in \mathcal{B}$, with $\operatorname{diam}(U)>0, d_{k}(U) \leq \frac{c^{1+s} \cdot 2^{-k}}{\operatorname{diam}(U)^{s}}$.

\section{Proof of Claim 3.12 ,}

We prove by induction on $n-m$ that for every $n, m \in \mathbb{N}$ with $m<n, U \subseteq V$ with $\operatorname{diam}(U)>0, U \in \mathcal{B}_{n}$ and $V \in \mathcal{B}_{m}$,

$$
d_{k}(U) \leq \frac{d_{k}(V) \operatorname{diam}(V)^{s}}{\operatorname{diam}(U)^{s}} .
$$

By the definition of supergale, if $U \in \mathcal{B}_{n}, d_{k}(U) \leq \frac{d_{k}\left(U^{\prime}\right) \operatorname{diam}\left(U^{\prime}\right)^{s}}{\operatorname{diam}(U)^{s}}$ for $U^{\prime} \in$ $\mathcal{B}_{n-1}$ with $U \subseteq U^{\prime}$. By induction $d_{k}\left(U^{\prime}\right) \leq \frac{d_{k}(V) \operatorname{diam}(V)^{s}}{\operatorname{diam}\left(U^{\prime}\right)^{s}}$ and therefore $d_{k}(U) \leq$ $\frac{d_{k}(V) \operatorname{diam}(V)^{s}}{\operatorname{diam}(U)^{s}}$.

For every $W \in \mathcal{B}_{0}$ with $\operatorname{diam}(W)>0$, we use the second part in the definition of $d_{k}$ and so $d_{k}(W) \leq \frac{c^{1+s} \cdot 2^{-k}}{\operatorname{diam}(W)^{s}}$.

Since for every $U \in \mathcal{B}$ there is a $W \in \mathcal{B}_{0}$ with $U \subseteq W$ we have that

$$
d_{k}(U) \leq \frac{d_{k}(W) \operatorname{diam}(W)^{s}}{\operatorname{diam}(U)^{s}} \leq \frac{c^{1+s} \cdot 2^{-k}}{\operatorname{diam}(U)^{s}} .
$$


We define next an $s$-supergale $d(U)=\sum_{k} 2^{k} d_{2 k}(U)$.

By Claim $3.12 d$ is well-defined.

By Claim 3.11 if $W \in \mathcal{D}_{k}, d(W) \geq 2^{k}$. Since for every $k, \mathcal{D}_{k} \subseteq \mathcal{B}$ is a cover of $A$, by Property 3.7 we have that $A \subseteq S^{\infty}[d]$ and $s \in \mathcal{G}(A)$.

For the other direction, let $s \in \hat{\mathcal{G}}(A)$. Then there exists an $s$-supergale $d$ such that $A \subseteq S^{\infty}[d]$.

For each $k \in \mathbb{N}$ let

$$
\mathcal{C}_{k}=\left\{U \mid \operatorname{diam}(U)>0, d(U)>2^{k} \cdot \sum_{W \in \mathcal{B}_{0}} d(W) \operatorname{diam}(W)^{s}\right\},
$$

let $\mathcal{D}_{k}=\left\{U \mid U \in \mathcal{C}_{k}\right.$ and no proper superset of $U$ is in $\left.\mathcal{C}_{k}\right\}$. Then, using Property 3.8. $\sum_{U \in \mathcal{D}_{k}} \operatorname{diam}(U)^{s} \leq 2^{-k}$.

Notice that for every $k, \mathcal{D}_{k}$ is a $2^{-k / s}$-cover of $S^{\infty}[d]$, so $\operatorname{dim}_{\mathrm{H}}(A) \leq s$. This completes our proof.

Notice that a characterization of Hausdorff dimension in terms of martingales (that is, 1-gales) that holds for Cantor space is not clear for the general case. $d(U)$ is an $s$-gale if and only if $d^{\prime}(U)=\operatorname{diam}(U)^{s-1} d(U)$ is a 1-gale, but it is not clear how to express $S^{\infty}[d]$ in terms of $d^{\prime}$.

For the examples in subsection 3.1 we obtain generalizations of the concept of gale used in previous effectivizations.

Example 3.13 For the set $\Sigma^{\infty}$ of all infinite sequences over a finite alphabet $\Sigma$ with the metric based on a positive and nonatomic Borel probability measure $\nu: \Sigma^{*} \rightarrow[0,1]$. (Generalization of [13]).

For $s \in[0, \infty)$, an $s$-supergale $d$ is a function $d: \Sigma^{*} \rightarrow[0, \infty)$ such that for every $n \in \mathbb{N}$, for every $w \in \Sigma^{*}$ with $|w|=n$ the following inequality holds

$$
d(w) \nu(w)^{s} \geq \sum_{a \in \Sigma} d(w a) \nu(w a)^{s}
$$

Example 3.14 For $m \in \mathbb{N}$, consider the set $\mathbb{R}^{m}$ with the metric based on a strongly positive Borel measure $\nu$. (Generalization of [13]).

For $s \in[0, \infty)$, an s-supergale $d$ is a function $d: \mathbb{Z}^{m} \times \mathbb{N}^{m} \times \mathbb{N} \rightarrow[0, \infty)$ such that for every $\mathbf{z} \in \mathbb{Z}^{m}, \mathbf{a} \in \mathbb{N}^{m}, n \in \mathbb{N}$ (with $a_{i}<2^{n}$ ), the following inequality holds

$$
\begin{array}{r}
d(\mathbf{z}, \mathbf{a}, n) \nu(I(\mathbf{z}, \mathbf{a}, n))^{s} \geq \\
\sum_{I(\mathbf{t}, \mathbf{b}, n+1) \subseteq I(\mathbf{z}, \mathbf{a}, n)} d(\mathbf{t}, \mathbf{b}, n+1) \nu(I(\mathbf{t}, \mathbf{b}, n+1))^{s} .
\end{array}
$$

Example 3.15 Let $h: \mathbb{N} \rightarrow \mathbb{N}-\{0,1\}, h^{\omega}=\Pi_{n \in \mathbb{N}}\{0,1, \ldots, h(n)-1\}$ with the metric based on a positive and nonatomic Borel probability measure $\nu$. (Generalization of [15]). 
For $s \in[0, \infty)$, an $s$-supergale $d$ is a function $d: h^{*} \rightarrow[0, \infty)$ such that for every $n \in \mathbb{N}$, for every $w \in h^{n}$ the following inequality holds

$$
d(w) \nu(w)^{s} \geq \sum_{a \in \Sigma} d(w a) \nu(w a)^{s} .
$$

Example 3.16 $P_{n}(0,1)$ is the set of polynomials with real coefficients and degree less than or equal to $n$, together with the metric $d(f, g)=\|f-g\|_{\infty}$.

For $s \in[0, \infty)$, an s-supergale $d$ is a function $d: \mathbb{Z}^{n+1} \times \mathbb{N}^{n+1} \times \mathbb{N} \rightarrow[0, \infty)$ such that for every $\mathbf{z} \in \mathbb{Z}^{n+1}, \mathbf{a} \in \mathbb{N}^{n+1}, k \in \mathbb{N}$ (with $a_{i}<2^{k}$ ), the following inequality holds

$$
d(\mathbf{z}, \mathbf{a}, k) \geq 2^{-s} \sum_{D P(\mathbf{t}, \mathbf{b}, k+1) \subseteq D P(\mathbf{z}, \mathbf{a}, k)} d(\mathbf{t}, \mathbf{b}, k+1) .
$$

\section{Constructive dimension}

In this section we effectivize Hausdorff dimension by considering constructive dimension. We consider spaces that have computable nice covers (defined below) and define constructive dimension in them.

Then we characterize constructive dimension in terms of Kolmogorov complexity using the concept of Kolmogorov complexity of $x \in X$ at precision $r \in \mathbb{N}$ inspired by [13. This characterization, together with the absolute stability proven below allows a full Theory of Information view of Hausdorff dimension in some general metric spaces.

Definition. Let $X$ be a metric space with a nice cover $\left(\mathcal{B}_{n}\right)_{n \in \mathbb{N}}$. Let $\Sigma$ be finite and $\delta: \Sigma^{*} \rightarrow \mathcal{B}$ be surjective. We say that $\left(X,\left(\mathcal{B}_{n}\right), \delta\right)$ has a computable nice cover if the following hold,

(B5) (Computable diameter) diam $\circ \delta$ is a computable function.

(B6) (Computable cover) The function

$$
P: \Sigma^{*} \times \mathbb{N} \rightarrow \Sigma^{*}
$$

defined by $P(w, n)=<w_{1}, \ldots, w_{k}>$ for $\delta(w) \in \mathcal{B}_{n}$, such that $\left\{V \mid V \in \mathcal{B}_{n+1}, V \subseteq \delta(w)\right\}=\left\{\delta\left(w_{1}\right), \ldots, \delta\left(w_{k}\right)\right\}$ is a computable function.

For the rest of this section we fix a space $\left(X,\left(\mathcal{B}_{n}\right), \delta\right)$ with a computable nice cover and omit $\left(\mathcal{B}_{n}\right), \delta$ when referring to $X$.

Definition. Let $d$ be a supergale. Then $d$ is contructive if $d \circ \delta$ is lower semicomputable and $\sum_{U \in \mathcal{B}_{0}} d(U) \operatorname{diam}(U)^{s}$ is a computable number.

Definition. Let $A \subseteq X$,

$$
\hat{\mathcal{G}}_{\text {constr }}(A)=\left\{s \mid \text { there is a constructive } s \text {-supergale } d \text { with } A \subseteq S^{\infty}[d]\right\} .
$$


Definition. Let $A \subseteq X$. We define the constructive dimension of $A$ as $\operatorname{cdim}(A)=\inf \hat{\mathcal{G}}_{\text {constr }}(A)$.

For each $x \in X$ we denote with $\operatorname{cdim}(x)$ the constructive dimension of the singleton set, that is, $\operatorname{cdim}(x)=\operatorname{cdim}(\{x\})$.

Let us briefly comment on the importance of the choice of nice cover when effectivizing a dimension. The classical definition of Hausdorff dimension is invariant under the choice of nice cover as our characterization in Theorem 3.9 proves. In the case of effective dimension, however, this invariance does not generally hold (the choice of $\left(\mathcal{B}_{n}\right), \delta$ can be relevant) and this fact is very meaningful. For instance in the case of Finite-State dimension in $\mathbb{R}^{n}$, that is, restriction to gales that can be computed by Finite State Automata (done for Cantor space in 2]), it matters whether we use dyadic intervals, triadic intervals, etc and this is related to the existence of normal sequences that are not absolutely normal [2]. On the other hand certain invariance properties are known for constructive and polynomial-time dimension 8 .

We next look at the compression characterization of constructive dimension. Notice that the definition of Kolmogorov complexity at a certain precision is more involved than in the Cantor case (or even slightly more than in the Euclidean case) when there is no Borel measure.

Constructive dimension can be characterized in terms of Kolmogorov complexity as follows. Let $\mathrm{K}(w)$ denote the usual self-delimiting Kolmogorov complexity of $w \in \Sigma^{*}$.

Definition. Let $x \in X$, let $r \in \mathbb{N}$. The Kolmogorov complexity of $x$ at precision $r$ is

$$
\mathrm{K}_{r}(x)=\inf \left\{\mathrm{K}(w) \mid x \in \delta(w), \operatorname{diam}(\delta(w)) \leq 2^{-r}\right\},
$$

with $\mathrm{K}_{r}(x)=\infty$ if not such $w$ exists.

Theorem 4.1 Let $X$ be a metric space with a computable nice cover. Let $Z \subseteq$ $X$,

$$
\operatorname{cdim}(Z)=\sup _{x \in Z} \liminf _{r} \frac{\mathrm{K}_{r}(x)}{r} .
$$

\section{Proof.}

The first direction uses semicomputability and additivity properties of prefix Kolmogorov complexity. Let

Let $s, s^{\prime}, s^{\prime \prime}$ be rational numbers such that $s>s^{\prime}>s^{\prime \prime}>\sup _{x \in Z} \liminf \operatorname{in}_{r} \frac{\mathrm{K}_{r}(x)}{r}$.

$$
A=\left\{w \mid \mathrm{K}(w) \leq-s^{\prime} \log (\operatorname{diam}(\delta(w)))\right\} .
$$

Then $A$ is computably enumerable.

We define $d$ as follows, let $U \in \mathcal{B}_{n}$ with $\operatorname{diam}(U)>0$,

$$
d(U)=\sum_{V \subseteq U, V \in \delta(A) \cap \mathcal{B}_{\geq n}} \frac{\operatorname{diam}(V)^{s^{\prime}}}{\operatorname{diam}(U)^{s}} .
$$


$d$ is well defined since $\sum_{V \in \delta(A)} \operatorname{diam}(V)^{s^{\prime}} \leq \sum_{w} 2^{-\mathrm{K}(w)}<\infty . \quad d$ is constructible by property (B6).

$d$ is an $s$-supergale since for $W \in \mathcal{B}_{n-1}$,

$$
\begin{gathered}
\sum_{U \subseteq W, U \in \mathcal{B}_{n}} d(U) \operatorname{diam}(U)^{s}=\sum_{U \subseteq W, U \in \mathcal{B}_{n}} \sum_{V \subseteq U, V \in \delta(A) \cap \mathcal{B}_{\geq n}} \operatorname{diam}(V)^{s^{\prime}} \leq \\
\leq \sum_{V \subseteq W, V \in \delta(A) \cap \mathcal{B}_{\geq n-1}} \operatorname{diam}(V)^{s^{\prime}}=d(W) \operatorname{diam}(W)^{s},
\end{gathered}
$$

where the last property follows from property (A2).

If $U \in \delta(A)$ then $d(U) \geq \operatorname{diam}(U)^{s^{\prime}-s}$. Let $x \in Z$. Since $\mathrm{K}_{r}(x)<r s^{\prime \prime}$ for infinitely many $r$, for those $r$ there is a $w_{r}$ with $\mathrm{K}\left(w_{r}\right) \leq r s^{\prime \prime}, x \in \delta\left(w_{r}\right)$ and $\operatorname{diam}\left(\delta\left(w_{r}\right)\right) \leq 2^{-r}$. Therefore $w_{r} \in A$ and $d\left(\delta\left(w_{r}\right)\right) \geq \operatorname{diam}\left(\delta\left(w_{r}\right)\right)^{s^{\prime}-s} \geq$ $2^{r\left(s-s^{\prime}\right)}$.

Therefore for each $x \in Z$ there is $\left(\delta\left(w_{r}\right)\right) \subseteq \mathcal{B}$ with $\limsup _{r} d\left(\delta\left(w_{r}\right)\right)=\infty$ and by Property $3.7 x \in S^{\infty}[d]$. Therefore $Z \subseteq S^{\infty}[d]$.

For the other direction, let $s>\operatorname{cdim}(Z)$. Let $d$ be a constructive $s$-supergale such that $Z \subseteq S^{\infty}[d]$. For each $k \in \mathbb{N}$, let

$$
A_{k}=\left\{w \mid d(\delta(w)) \geq 2^{k}\left(\sum_{W \in \mathcal{B}_{0}} d(W) \operatorname{diam}(W)^{s}\right)\right\} .
$$

Then by Property 3.8, for each $r \in \mathbb{N}$ and for every $\mathcal{E} \subseteq \mathcal{B}$ such that all sets in $\mathcal{E}$ are incomparable, the number of $w \in A_{k} \cap \delta^{-1}(\mathcal{E})$ such that $\operatorname{diam}(\delta(w))>2^{-r-1}$ is at most $2^{-k+r s+s}$. Also by Property 3.8 if $w \in A_{k}$ then $\operatorname{diam}(\delta(w)) \leq 2^{-k / s}$.

Fix $k \in \mathbb{N}$. We enumerate all strings $u$ in $A_{k}$ and include $\delta(u)$ in $\mathcal{E}^{k}$ for the strings such that $\delta(u)$ is incomparable with all sets previously included in $\mathcal{E}^{k}$.

For $w \in A_{k} \cap \delta^{-1}\left(\mathcal{E}^{k}\right)$ there is an $r \geq\lceil k / s-1\rceil$ with $2^{-r-1}<\operatorname{diam}(\delta(w)) \leq$ $2^{-r}$, and $\mathrm{K}(w) \leq r s+s-k+O(\log k)+O(\log r)$.

Since $x \in S^{\infty}[d]$ for every $k$ there is $w \in A_{k} \cap \delta^{-1}\left(\mathcal{E}^{k}\right)$ such that $x \in \delta(w)$ and therefore

$$
\liminf _{r} \frac{\mathrm{K}_{r}(x)}{r} \leq \frac{r s+s-k+O(\log k)+O(\log r)}{r} \leq s .
$$

Taking the supremum over all $x \in Z$ we have proven our Theorem.

Corollary 4.2 Let $X$ be a metric space with a computable nice cover. Let $x \in X$,

$$
\operatorname{cdim}(x)=\liminf _{r} \frac{\mathrm{K}_{r}(x)}{r} .
$$

As a corollary we have the property of total stability of constructive dimension (see [11] for the corresponding version in Cantor space).

Corollary 4.3 Let $X$ be a metric space with a computable nice cover. Let $A \subseteq X$. Then

$$
\operatorname{cdim}(A)=\sup _{x \in A} \operatorname{cdim}(x) .
$$


We finish by presenting the constructive dimension of the spaces in all previous examples. Notice that all the examples in subsection 3.1 have computable nice covers.

Example 4.4 For the set $\Sigma^{\infty}$ of all infinite sequences over a finite alphabet $\Sigma$ with the metric based on a positive and nonatomic Borel probability measure $\nu: \Sigma^{*} \rightarrow[0,1]$. (Generalization of [13]).

Constructive supergales are constructive functions $d: \Sigma^{*} \rightarrow[0, \infty)$ that fulfill the supergale inequality.

For $x \in \Sigma^{\infty}$, the Kolmogorov complexity of $x$ at precision $r$ is

$$
\mathrm{K}_{r}(x)=\inf \left\{\mathrm{K}(w) \mid w \sqsubseteq x, \nu(w) \leq 2^{-r}\right\} .
$$

Example 4.5 For the set $\mathbb{R}^{m}(m \in \mathbb{N})$ with the metric based on a strongly positive Borel measure $\nu$. (Generalization of [13]).

Constructive supergales are constructive functions $d: \mathbb{Z}^{m} \times \mathbb{N}^{m} \times \mathbb{N} \rightarrow[0, \infty)$ that fulfill inequality (2).

For $x \in \mathbb{R}^{m}$, the Kolmogorov complexity of $x$ at precision $r$ is

$$
\begin{aligned}
\mathrm{K}_{r}(x)=\inf \{\mathrm{K}(w) \quad \mid \quad & w=<\mathbf{z}, \mathbf{a}, n>, \mathbf{z} \in \mathbb{Z}^{m}, \mathbf{a} \in \mathbb{N}^{m}, n \in \mathbb{N}, a_{i}<2^{n}, x \in I(\mathbf{z}, \mathbf{a}, n), \\
& \left.\nu(I(\mathbf{z}, \mathbf{a}, n)) \leq 2^{-r}\right\} .
\end{aligned}
$$

Example 4.6 Let $h: \mathbb{N} \rightarrow \mathbb{N}-\{0,1\}, h^{\omega}=\Pi_{n \in \mathbb{N}}\{0,1, \ldots, h(n)-1\}$ with the metric based on a positive and nonatomic Borel probability measure $\nu$. (Generalization of [15]).

Constructive supergales are constructive functions $d: h^{*} \rightarrow[0, \infty)$ that fulfill the supergale inequality.

For $x \in h^{\omega}$, the Kolmogorov complexity of $x$ at precision $r$ is

$$
\mathrm{K}_{r}(x)=\inf \left\{\mathrm{K}(w) \mid w \text { codifies } y \in h^{*}, y \sqsubseteq x, \nu(y) \leq 2^{-r}\right\} \text {. }
$$

Example $4.7 P_{n}(0,1)$ is the set of polynomials with real coefficients and degree less than or equal to $n$, together with the metric $d(f, g)=\|f-g\|_{\infty}$.

Constructive supergales are constructive functions $d: \mathbb{Z}^{n+1} \times \mathbb{N}^{n+1} \times \mathbb{N} \rightarrow$ $[0, \infty)$ that fulfill inequality (3).

For $f \in P_{n}(0,1)$, the Kolmogorov complexity of $f$ at precision $r$ is

$$
\begin{aligned}
\mathrm{K}_{r}(f)=\inf \{\mathrm{K}(w) \quad \mid & w=<\mathbf{z}, \mathbf{a}, k>, \mathbf{z} \in \mathbb{Z}^{n+1}, \mathbf{a} \in \mathbb{N}^{n+1}, k \in \mathbb{N}, a_{i}<2^{k}, \\
& f \in \operatorname{DP}(\mathbf{z}, \mathbf{a}, k), k \geq r+\log (n+1)\} .
\end{aligned}
$$

\section{$5 \quad$ Further directions}

This paper intends to give an initial view of effective dimension on arbitrary metric spaces. A number of issues have not been addressed here including the definition of resource-bounded dimension for resource-bounds other than lower semicomputability and the role of different (computable) nice covers in effectivization and conditions for their equivalence within it. 


\section{Acknowledgment}

We thank Donald Stull for suggesting example 3.6 .

\section{References}

[1] J. Cai and J. Hartmanis. On Hausdorff and topological dimensions of the Kolmogorov complexity of the real line. Journal of Computer and Systems Sciences, 49:605-619, 1994.

[2] J. J. Dai, J. I. Lathrop, J. H. Lutz, and E. Mayordomo. Finite-state dimension. Theoretical Computer Science, 310:1-33, 2004.

[3] R. G. Downey and D. R. Hirschfeldt. Algorithmic randomness and complexity. Springer-Verlag, 2010.

[4] K. Falconer. Fractal Geometry: Mathematical Foundations and Applications. John Wiley \& sons, 2003.

[5] P. Gács. Uniform test of algorithmic randomness over a general space. Theoretical Computer Science, 341:91-137, 2005.

[6] F. Hausdorff. Dimension und äußeres Maß. Math. Ann., 79:157-179, 1919.

[7] J. M. Hitchcock, J. H. Lutz, and E. Mayordomo. The fractal geometry of complexity classes. SIGACT News Complexity Theory Column, 36:24-38, 2005.

[8] J.M. Hitchcock and E. Mayordomo. Base invariance of feasible dimension. Information Processing Letters, 113:546-551, 2013.

[9] M. Hoyrup and C. Rojas. Computability of probability measures and martin-löf randomness over metric spaces. Information and Computation, 207:830-847, 2009.

[10] J. H. Lutz. Dimension in complexity classes. SIAM Journal on Computing, 32(5):1236-1259, 2003.

[11] J. H. Lutz. The dimensions of individual strings and sequences. Information and Computation, 187(1):49-79, 2003.

[12] J. H. Lutz and N. Lutz. Algorithm information, plane kakeya sets, and conditional dimension. submitted.

[13] J.H. Lutz and E. Mayordomo. Dimensions of points in self-similar fractals. SIAM Journal on Computing, 38:1080-1112, 2008.

[14] E. Mayordomo. Effective fractal dimension in algorithmic information theory. In New Computational Paradigms: Changing Conceptions of What is Computable, pages 259-285. Springer-Verlag, 2008. 
[15] J. Miller and N. Greenberg. Diagonally non-recursive functions and effective hausdorff dimension. Bulletin of the London Mathematical Society, 43:636654, 2011.

[16] K. Miyabe. Algorithmic randomness over general spaces. Math. Log. Quart., 60:184-204, 2014.

[17] B. Ya. Ryabko. Coding of combinatorial sources and hausdorff dimension. Soviets Mathematics Doklady, 30:219-222, 1984.

[18] B. Ya. Ryabko. Noiseless coding of combinatorial sources. Problems of Information Transmission, 22:170-179, 1986.

[19] S.G. Simpson. Symbolic dynamics: entropy $=$ dimension $=$ complexity. Theory of Computing Systems, 56:527-543, 2015.

[20] L. Staiger. Kolmogorov complexity and Hausdorff dimension. Information and Computation, 103:159-94, 1993.

[21] L. Staiger. A tight upper bound on Kolmogorov complexity and uniformly optimal prediction. Theory of Computing Systems, 31:215-29, 1998. 